\title{
The Use of Positron Emission Tomography in the Diagnosis of Prosthetic Arteriovenous Hemodialysis Graft Infection: A Case Study
}

\author{
Bertrand Saint-Lebes ${ }^{1 *}$, Celine Dubuis ${ }^{2}$, Sebastien Deglise ${ }^{2}$, \\ François Saucy $^{2}$, Jean-Marc Corpataux ${ }^{2}$ \\ ${ }^{1}$ Department of Vascular Surgery, University Hospital, Toulouse, France \\ ${ }^{2}$ Department of Vascular Surgery, Centre Hospitalier Universitaire Vaudois (CHUV), University Hospital, Lausanne, Switzerland \\ Email: saintlebes.b@chu-toulouse.fr
}

Received January 14, 2014; revised February 9, 2014; accepted February 16, 2014

Copyright (C) 2014 Bertrand Saint-Lebes et al. This is an open access article distributed under the Creative Commons Attribution License, which permits unrestricted use, distribution, and reproduction in any medium, provided the original work is properly cited. In accordance of the Creative Commons Attribution License all Copyrights (C) 2014 are reserved for SCIRP and the owner of the intellectual property Bertrand Saint-Lebes et al. All Copyright (C) 2014 are guarded by law and by SCIRP as a guardian.

\begin{abstract}
Patients on chronic hemodialysis (HD) required efficient arterio-venous access. Different types of vascular access are commonly used, such as native arteriovenous (AV) fistula, prosthetic AV graft, central venous catheter, or temporary and tunnelled cuffed catheters. Vascular access-related morbidity remains the major issue for patients requiring long-term therapy. Vascular access infection constitutes the most challenging and life-threatening complication of vascular access and causes significant morbidity, loss of access and mortality. Vascular access infection is defined as local signs at the vascular access site or a positive blood culture with no know source other than the vascular access. The critical issue in the management of AV graft infection is the need to remove the infection and to maintain HD access with reduced morbidity. The diagnosis of infected AV graft is not always easy, especially with low-grade infection or atypical presentation. Fluorodeoxyglucose positron emission tomography (FDG-PET) is an imaging method that uses a radioactively labelled tracer (18 fluorodeoxy glucose) which is taken up by tissue with high metabolic activity, such as neoplastic and inflammatory lesions. FDG-PET has been proposed to visualise localisation of infection in patients with suspected prosthetic graft infection. We report a case of methicilin resistant staphylococcus aureus (MRSA) hemodialysis access infection without local inflammatory signs diagnosed on the PET-CT.
\end{abstract}

\section{KEYWORDS}

Hemodialysis Access; Graft Infection; PET-CT

\section{Introduction}

Patients on chronic hemodialysis (HD) required efficient arterio-venous access. Achieving a durable and reliable vascular access is an important determinant of successful therapy. Different type of vascular access are commonly used, such as native arteriovenous (AV) fistula, prosthetic AV graft, central venous catheter, or temporary and tunnelled cuffed catheters. Native AV fistula has been considered the most favourable in terms of function, duration and absence of complications [1,2]. Vascular access - related morbidity remains the major issue for patients requiring

\footnotetext{
*Corresponding author.
}

long-term therapy.

Vascular access infection can result from contamination during the construction of the access, from the repeated use of the access or intervention to prolong the use of access. It constitutes the most challenging and lifethreatening complication of vascular access and causes significant morbidity, loss of access and mortality. After cardiovascular events, infections among dialysis patients account for $15 \%$ - $36 \%$ of all deaths and for about $20 \%$ of admissions [3].

The type of vascular access is a well-recognized risk factor for infection as demonstrated in several studies [46]. AV fistula have the lowest infection rate, followed by 
AV graft, tunnelled cuffed catheter and temporary catheter. AV graft have an incidence of infection from $11 \%$ $35 \%$ [7] with complications such as thrombosis, perigraft hematoma and pseudoaneurysm that cause access loss in $60 \%$ while infection causes graft failure in $35 \%$ [8].

Vascular access infection is defined as local signs at the vascular access site or a positive blood culture with no know source other than the vascular access. The critical issue in the management of AV graft infection is the need to remove the infection and to maintain HD access with reduced morbidity. Treatment usually involves intravenous antibiotics and graft excision. Staphylococcus aureus is the most common organism found in case of AVFistula and AV graft [9].

The diagnosis of infected AV graft is not always easy, especially with low-grade infection or atypical presentation. In addition to clinical signs, evaluation of infection parameters and blood cultures should be done. To assess the extent of the involvement ultrasonography examination, computed tomography scanning, magnetic resonance imaging can be performed, however the predictive value for vascular prosthetic infection with either one of theses diagnostic tools is relatively low [10].

Fluorodeoxyglucose positron emission tomography (FDG-PET) is an imaging method that uses a radioactively labelled tracer (18 fluorodeoxy glucose) which is taken up by tissue with high metabolic activity, such as neoplastic and inflammatory lesions. (Wahl R. Principles of cancer imaging with fluorodeoxyglucose. In: Wahl R., Buchanan J (eds). Principles and Practice of Positron Emission Tomography. Philadelphia, PA: Lippincott Williams \& Wilkins, 2002; 100 - 110). At the beginning, the observation that FDG accumulates at the sites of infection and inflammation has been considered as a potential source of false-positive results in the diagnosis of cancer. Actually FDG-PET is increasingly used to assess patients with infectious and inflammatory disorders. FDG-PET has been proposed to visualise localisation of infection in patients with suspected prosthetic graft infection $[11,12]$.

We report a case of methicilin resistant staphylococcus aureus (MRSA) hemodialysis access infection without local inflammatory signs diagnosed on the PET-CT.

\section{Case Report}

We present the case of an 81-year-old patient on dialysis since 2008 for a terminal renal insufficieny due to a diabetic and hypertensive nephropathy. Among the relevant comorbidities he suffers from COPD, a hypertensive cardiopathy with left ventricule hypertrophy, and a metabolic syndrome.

He began haemodialysis on a native AVFistula, who was proximalised and finally replaced by a prosthetic
ePTFE AVFistula on the left arm.

While treated with ciproxin for an MRSA urinary tract infection, he developped a painful swollen right wrist with an inflammatory syndrome (CRP 70, leucocyts 12) without fever. The first hypothesis was a septic arthritis of the wrist who could not be exculded because of non conclusive puncture. In the meanwhile all the hemocultures became positive for a MRSA. To exculde an infection of the AVF, an Ultra Sound was performed and showed partially thrombosed small aneurysms without collection or signs of infection. A transthoracic ultrasound excludes an endocarditis.

The appreciation at the time was a MRSA bacteriemia of urinary origin with a suspicion of septic arthritis. The patient received vancomycin adapted to his renal function and it was decided to do a clinical follow-up.

In front of a persistant bacteriemia despite the target Antibiotic during 6 days, we decided to performed a PET CT that reveals the presence of 3 focal FDG uptake on the arteriovenous graft (Figures 1 and 2).

\section{Results}

The patient was treated with total graft exision. No pus was found peroperatively and the prosthesis was well incorporated without anastomotic pseudoaneurysms. On the prosthetic loop, two aneurysms containing thrombus were found, according to the ultrasound.

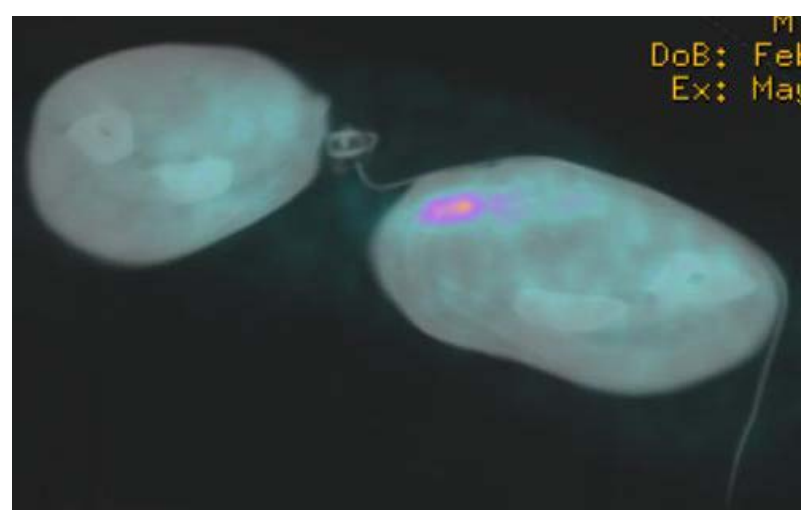

Figure 1. PET CT: Focal FDG uptake on the arteriovenous graft.

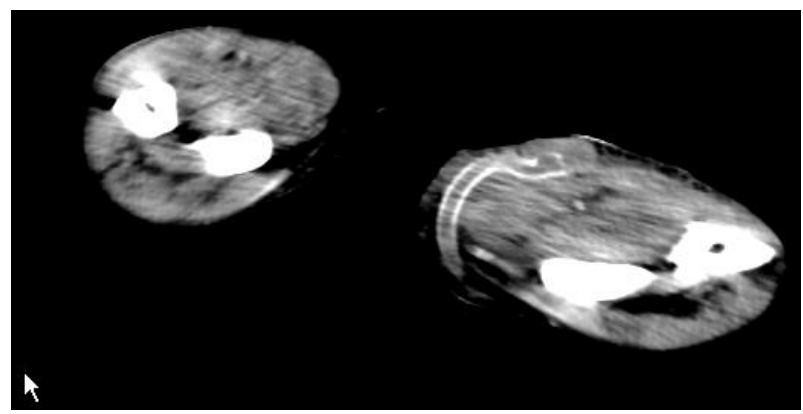

Figure 2. CT: Arteriovenous graft. 
After negative blood culture, a new prosthetic AV fistula was then created on the right forearm together with a cuffed tunnelled catheter.

Microbiological exam of the excised prosthesis was positive for a MRSA. The vancomycin was continued for a month.

We encountered no problem regarding the wound healing and the patient was discharged 15 days later, with normalized laboratory tests and a functioning right arm fistula.

\section{Discussion}

Prosthetic bypass graft is an irreplaceable material in patients with no suitable superficial veins. With his high morbidity incidence rate, vascular access infection has a significant impact on the morbidity and mortality in HD patients. Therefore early and reliable diagnoses are necessary to provide adequate treatment and avoid further complications, such as anastomotic bleeding, sepsis or even death.

Diagnosis of graft infection is usually established according to clinical findings. However, some cases have a subtle and non specific clinical presentation, with nonspecific conventional imaging findings, that make the diagnosis difficult.

The gold standard for the diagnosis of infected vascular prosthesis is still the CT scan, which has good diagnostic accuracy in patients with advanced graft infection, but fails in low-grade infection. Fiorani [13] et al. described that CT is accurate in diagnosing advanced graft infection such as periprosthetic abscess or in case of aortoenteric fistula, but not in low-grade infection. The advantages offered by FDG-PET include the completion of the examination within a short period of time, high interobserver agreement, low radiation dose and high specificity for assessment of cellular metabolic activity of the inflammatory process rather than assessment of perfusion or edema as indirect evidence for cellular activity [14]. FDG-PET alone has a high sensitivity for detecting vascular graft infection but it lacks specificity when compared to CT [15]. Adding CT to PET can increase specificity when evaluating graft infection.

Some published series have evaluated the usefullness of FDG-PET in the diagnosis of vascular graft infection. Fukuchi et al. [15] compared the efficacy of FDG-PET to CT in patients with suspected aortic graft infection. They conclued that identification of the characteristic FDG uptake pattern (diffuse and intense) as a diagnostic criterion on FDG-PET is superior to those on CT. Keidar et al. [16] assess the performance of PET/CT for the diagnosis of an infectious process and its localization to the vascular graft or to the adjacent soft tissue. Their conclusion was that FDG-PET/CT is a reliable noninvasive imaging modality for the diagnosis of vascular graft-related infec- tion. The precise anatomic localization of increased FDG uptake enables accurate differentiation between graft and soft-tissue infection.

\section{Conclusions}

Vascular hemodialysis graft infection (VHGI) is a severe complication. Therefore, early and reliable diagnoses are necessary to provide adequate treatment and avoid further complications, such as anastomotic bleeding orsepsis, which can results in limb loss or even death.

FDG-PET/CT is emerging as a promising tool to detect VHGI in patients. FDG-PET alone has a high sensitivity for VHGI, butitlacks specificity when compared to CT. Thus, adding CT to the PET camera can increase specificity when evaluating this complication.

No study describes the use of PET for diagnosis of an arteriovenous prosthetic fistula. However, similitude between the prosthesis suggests that FDG-PET/CT has the same value for AV graft than for limb bypass or aortic grafts. Further studies are mandatory to define the use of $\mathrm{PET} / \mathrm{CT}$ in clinical strategies more precisely and to assess its accuracy in the diagnosis of AV fistula infection.

\section{REFERENCES}

[1] III. NKF-K/DOQI, “Clinical, Practice Guidelines for Vascular Access: Update 2000,” American Journal of Kidney Diseases, Vol. 37, No. 1, 2001, pp. S137-S181. http://dx.doi.org/10.1016/S0272-6386(01)70007-8

[2] J. Ethier, et al., "Vascular Access Use and Outcomes: An International Perspective from the Dialysis Outcomes and Practice Patterns Study,” Nephrology Dialysis Transplantation, Vol. 23, No. 10, 2008, pp. 3219-3126. http://dx.doi.org/10.1093/ndt/gfn261

[3] J. A. Akoh, "Vascular Access Infections: Epidemiology, Diagnosis, and Management," Current Infectious Disease Reports, Vol. 13, No. 4, 2011, pp. 324-332. http://dx.doi.org/10.1007/s11908-011-0192-x

[4] P. Ponce, et al., "A Prospective Study on Incidence of Bacterial Infections in Portuguese Dialysis Units,” $\mathrm{Ne}$ phron Clinical Practice, Vol. 107, No. 4, 2007, pp. 133138. http://dx.doi.org/10.1159/000110033

[5] K. B. Stevenson, et al., "Epidemiology of Hemodialysis Vascular Access Infections from Longitudinal Infection Surveillance Data: Predicting the Impact of NKF-DOQI Clinical Practice Guidelines for Vascular Access," American Journal of Kidney Diseases, Vol. 39, No. 3, 2002, pp. 549-555. http://dx.doi.org/10.1053/ajkd.2002.31405

[6] L. A. Colville and A. H. Lee, "Retrospective Analysis of Catheter-Related Infections in a Hemodialysis Unit," Infection Control and Hospital Epidemiology, Vol. 27, No. 9, 2006, pp. 969-973.

http://dx.doi.org/10.1086/507821

[7] P. Bachleda, et al., "Infectious Complications of Arteriovenous ePTFE Grafts for Hemodialysis,” Biomedical Papers, Vol. 154, No. 1, 2010, pp. 13-19. http://dx.doi.org/10.5507/bp.2010.005 
[8] W. P. Schutte, et al., "Surgical Treatment of Infected Prosthetic Dialysis Arteriovenous Grafts: Total versus Partial Graft Excision,” The American Journal of Surgery, Vol. 193, No. 3, 2007, pp. 385-388.

http://dx.doi.org/10.1016/j.amjsurg.2006.09.028

[9] I. Saeed Abdulrahman, et al., "A Prospective Study of Hemodialysis Access-Related Bacterial Infections,” Journal of Infection and Chemotherapy, Vol. 8, No. 3, 2002, pp. 242-246.

http://dx.doi.org/10.1007/s10156-002-0184-8

[10] J. L. Bruggink, et al., "Accuracy of FDG-PET-CT in the Diagnostic Work-Up of Vascular Prosthetic Graft Infection," European Journal of Vascular and Endovascular Surgery, Vol. 40, No. 3, 2010, pp. 348-354. http://dx.doi.org/10.1016/j.ejvs.2010.05.016

[11] R. Kumar, et al., "Role of Modern Imaging Techniques for Diagnosis of Infection in the Era of 18F-Fluorodeoxyglucose Positron Emission Tomography,” Clinical Microbiology Reviews, Vol. 21, No. 1, 2008, pp. 209-224. http://dx.doi.org/10.1128/CMR.00025-07

[12] M. G. van der Vaart, et al., "Application of PET/SPECT Imaging in Vascular Disease," European Journal of
Vascular and Endovascular Surgery, Vol. 35, No. 5, 2008, pp. 507-513. http://dx.doi.org/10.1016/j.ejvs.2007.11.016

[13] P. Fiorani, et al., "Detection of Aortic Graft Infection with leukocytes Labeled with Technetium 99m-Hexametazime," Journal of Vascular Surgery, Vol. 17, No. 1, 1993, pp. 87-95.

http://dx.doi.org/10.1016/0741-5214(93)90012-B

[14] S. Basu, et al., "Positron Emission Tomography as a Diagnostic Tool in Infection: Present Role and Future Possibilities,” Seminars in Nuclear Medicine, Vol. 39, No. 1, 2009, pp. 36-51. http://dx.doi.org/10.1053/j.semnuclmed.2008.08.004

[15] K. Fukuchi, et al., "Detection of Aortic Graft Infection by Fluorodeoxyglucose Positron Emission Tomography: Comparison with Computed Tomographic Findings,” Journal of Vascular Surgery, Vol. 42, No. 5, 2005, pp. 919-925. http://dx.doi.org/10.1016/j.jvs.2005.07.038

[16] Z. Keidar, et al., "Prosthetic Vascular Graft Infection: The Role of 18F-FDG PET/CT," Journal of Nuclear Medicine, Vol. 48, No. 8, 2007, pp. 1230-1236. http://dx.doi.org/10.2967/jnumed.107.040253 